\title{
Nuclear structure investigations of ${ }^{253-255}$ Es by laser spectroscopy
}

\author{
S. Nothhelfer $\odot,{ }^{1,2,3}$ Th. E. Albrecht-Schönzart $\odot,{ }^{4}$ M. Block $\odot,{ }^{1,2,3}$ P. Chhetri $\odot,{ }^{2,3}$ \\ Ch. E. Düllmann $\odot,{ }^{1,2,3}$ J. G. Ezold $\odot,{ }^{5}$ V. Gadelshin, ${ }^{1,6}$ A. Gaiser $\odot,{ }^{4, *}$ F. Giacoppo, ${ }^{2,3}$ R. Heinke $\odot,{ }^{1, \dagger}$ T. Kieck $\odot,{ }^{1,2,3}$ \\ N. Kneip $\odot,{ }^{1}$ M. Laatiaoui $\odot,{ }^{1,2,3}$ Ch. Mokry, ${ }^{1,2}$ S. Raeder $\odot,{ }^{2,3}$ J. Runke, ${ }^{1,3}$ F. Schneider, ${ }^{1,2}$ J. M. Sperling, ${ }^{4}$ D. Studer $\odot,{ }^{1}$ \\ P. Thörle-Pospiech , ${ }^{1,2}$ N. Trautmann, ${ }^{1}$ F. Weber $\odot,{ }^{1}$ and K. Wendt ${ }^{1}$ \\ ${ }^{1}$ Johannes Gutenberg-Universität Mainz, 55099 Mainz, Germany \\ ${ }^{2}$ Helmholtz-Institut Mainz, 55099 Mainz, Germany \\ ${ }^{3}$ GSI Helmholtzzentrum für Schwerionenforschung GmbH, 64291 Darmstadt, Germany \\ ${ }^{4}$ Florida State University, 32306 Tallahassee, Florida, USA \\ ${ }^{5}$ Oak Ridge National Laboratory, Oak Ridge, 37831 Oak Ridge, Tennessee, USA \\ ${ }^{6}$ Ural Federal University, 620002 Yekaterinburg, Russia
}

(Received 23 July 2021; accepted 3 January 2022; published 4 February 2022)

\begin{abstract}
Laser resonance ionization spectroscopy was performed on the rare einsteinium isotopes ${ }^{253-255} \mathrm{Es}$ at the RISIKO mass separator in Mainz. With low sample sizes ranging down to femtograms, the prominent $352 \mathrm{~nm}$-ground-state transition was measured in all three einsteinium isotopes, and four additional groundstate transitions were measured in ${ }^{254}$ Es. Hyperfine-structure analysis resulted in assigned spin values of $I\left({ }^{254} \mathrm{Es}\right)=7$ and $I\left({ }^{255} \mathrm{Es}\right)=7 / 2$. From the extracted coupling constants, nuclear magnetic dipole moments of $\mu_{I}\left({ }^{254} \mathrm{Es}\right)=3.42(7) \mu_{N}$ and $\mu_{I}\left({ }^{255} \mathrm{Es}\right)=4.14(10) \mu_{N}$ as well as spectroscopic electric quadrupole moments of $Q_{s}\left({ }^{254} \mathrm{Es}\right)=9.6(1.2) \mathrm{eb}$ and $Q_{s}\left({ }^{255} \mathrm{Es}\right)=5.1(1.7) \mathrm{eb}$ were derived. Our value for ${ }^{254} \mathrm{Es}$ deviates from the value of $\left|\mu_{I}\left({ }^{254} \mathrm{Es}\right)\right|=4.35(41) \mu_{N}$ extracted from the angular anisotropy of $\alpha$-radiation emitted by ${ }^{254}$ Es.
\end{abstract}

DOI: 10.1103/PhysRevC.105.L021302

Introduction. The region of the heavy actinides marks the transition from heavy to superheavy elements, which owe their existence to nuclear shell effects. Shell closures have been identified in deformed superheavy nuclei at neutron numbers $N=152$ and $N=162[1,2]$. Their strength does not only vary with proton number $Z$ but also is sensitive to changes in nuclear shapes. The strength of the $N=152$ shell is maximal in fermium and weakens for lighter and heavier elements [3]. As this shell closure is accessible in the heaviest actinides, a detailed survey of their nuclear structure evolution is thus important to understand the underlying nuclear force and the properties of heavier nuclei, e.g., those of the superheavy elements. To this end, laser spectroscopy can contribute by providing access to changes in nuclear mean-square charge radii, nuclear spins, magnetic dipole moments $\mu_{I}$, and spectroscopic electric quadrupole moments $Q_{s}[4,5]$. These latter observables provide information about the shape, deformation, and single-particle nuclear configurations. Einsteinium $\left({ }_{99} \mathrm{Es}\right)$ and fermium $\left({ }_{100} \mathrm{Fm}\right)$ are

\footnotetext{
*Present address: Chemical Sciences Division, Lawrence Berkeley National Laboratory, Berkeley, California 94720, USA.

${ }^{\dagger}$ Present address: CERN, CH-1211 Geneva 23, Switzerland.
}

Published by the American Physical Society under the terms of the Creative Commons Attribution 4.0 International license. Further distribution of this work must maintain attribution to the author(s) and the published article's title, journal citation, and DOI. the heaviest elements that can be produced in macroscopic quantities. Starting from, e.g., curium as target material, $\mu \mathrm{g}$-quantities of einsteinium [6] are available after neutron activation in high-flux research reactors like the High Flux Isotope Reactor (HFIR) at Oak Ridge National Laboratory (ORNL) [7], reaching isotopes beyond the deformed shell closure at $N=152$ close to the line of $\beta$-stability, see Fig. 1. Only few properties are reported for einsteinium, despite its availability in macroscopic quantities. ${ }^{253} \mathrm{Es}$ was previously studied with optical spectroscopy using $\mu$ g-samples in discharge lamps and Fourier-transform spectrographs, yielding atomic transitions and hyperfine structure (HFS) splittings $[10,11]$. The latter allowed assigning the nuclear spin to $I=7 / 2$ and deducing the nuclear moment. A subsequent and more accurate measurement of the ground-state HFS of ${ }^{253}$ Es via atomic-beam magnetic-resonance (ABMR) resulted in $\mu_{I}=4.10(7) \mu_{N}$ and $Q_{s}=6.7(8) e b$ [12]. Embedded in an iron crystal lattice, ${ }^{254}$ Es was studied using the angular anisotropy of its $\alpha$-particle emission. Assuming $I=7$, taken from a tentative assignment from decay spectroscopy, $\left.\mid \mu_{I}{ }^{254} \mathrm{Es}\right) \mid=4.35(41) \mu_{N}$ was obtained [13-15]. The isomer ${ }^{254 \mathrm{~m}} \mathrm{Es}\left(T_{1 / 2}=39 \mathrm{~h}\right)$ was investigated and a nuclear spin of $I=2$ assigned [12].

A prerequisite for laser spectroscopy of heavy elements like einsteinium is the knowledge of suitable atomic transitions $[16,17]$. For neutral einsteinium, only 31 atomic levels have been reported $[17,18]$. In 1999, laser resonance ionization spectroscopy using a hot filament TOF technique on ${ }^{254} \mathrm{Es}$ was performed determining the first ionization potential (IP) to $51358(2) \mathrm{cm}^{-1}$ [18]. 


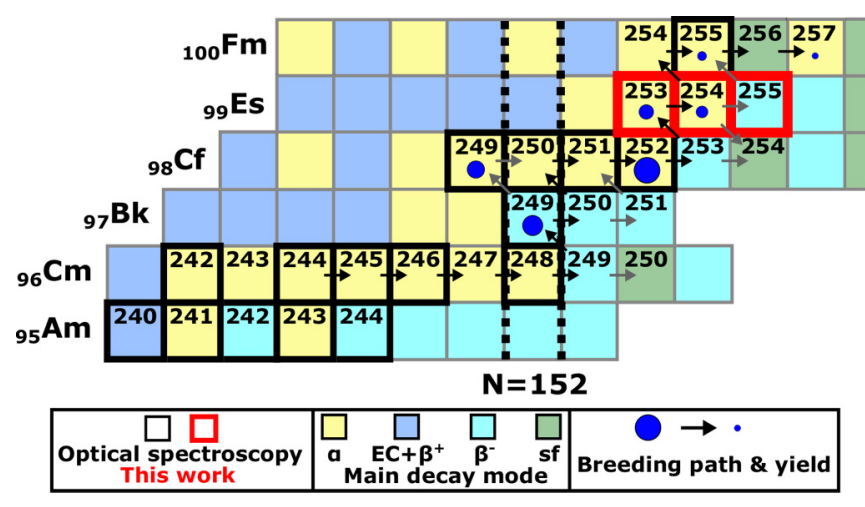

FIG. 1. Excerpt of the chart of nuclides. Indicated are nuclides investigated by optical spectroscopy $[5,8]$ and the breeding path for the Es isotopes starting from a mixed $\mathrm{Cm}$ target as given in $[6,7,9]$.

Resonance ionization spectroscopy (RIS) inside a hot cavity with a high-power Ti:sapphire (Ti:Sa) laser system is a highly efficient and sensitive method [16,20,21], enabling investigations on fg-quantities of Es isotopes, as reported in this work.

Experiment. An einsteinium sample was produced at ORNL in the HFIR [7], where four targets, each containing about $6.3 \mathrm{~g}$ of mixed curium isotopes $\left({ }^{244-248} \mathrm{Cm}\right)$, were irradiated by thermal neutrons, initiating nuclear transmutation in a series of neutron captures followed by $\beta^{-}$decays [22]. After irradiation, the targets were allowed to cool for 90 days to eliminate short-lived fission and activation products before being dissolved and chemically separated into fractions containing individual elements in the hot cells at the Radiochemical Engineering Development Center [6]. A sample containing $\approx 2 \mathrm{ng}{ }^{253} \mathrm{Es}, \approx 4 \mathrm{ng}{ }^{254} \mathrm{Es}$, and $\approx 4 \mathrm{pg}{ }^{255} \mathrm{Es}$ was dried and shipped to the Institute of Nuclear Chemistry at Johannes Gutenberg University Mainz (JGU). There, it was dissolved in $0.1 \mathrm{M}$ nitric acid. Three $3 \mu \mathrm{L}$-Aliquots containing about $10^{10}$ atoms of ${ }^{254}$ Es each were placed on a zirconium foil $[23,24]$ and evaporated to dryness.

The experiment was performed at the RISIKO mass separator [19] at JGU's Institute of Physics as shown in Fig. 2. The foil was folded to fully enclose the ${ }^{253-255}$ Es sample and

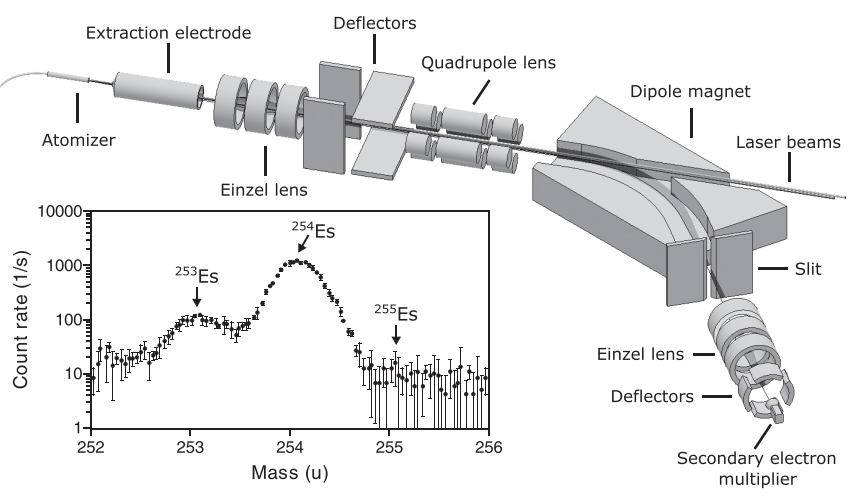

FIG. 2. Schematic overview of the RISIKO mass separator. For a more detailed description of the setup see [19]. The inset shows a mass spectrum of the laser-ionized einsteinium sample.

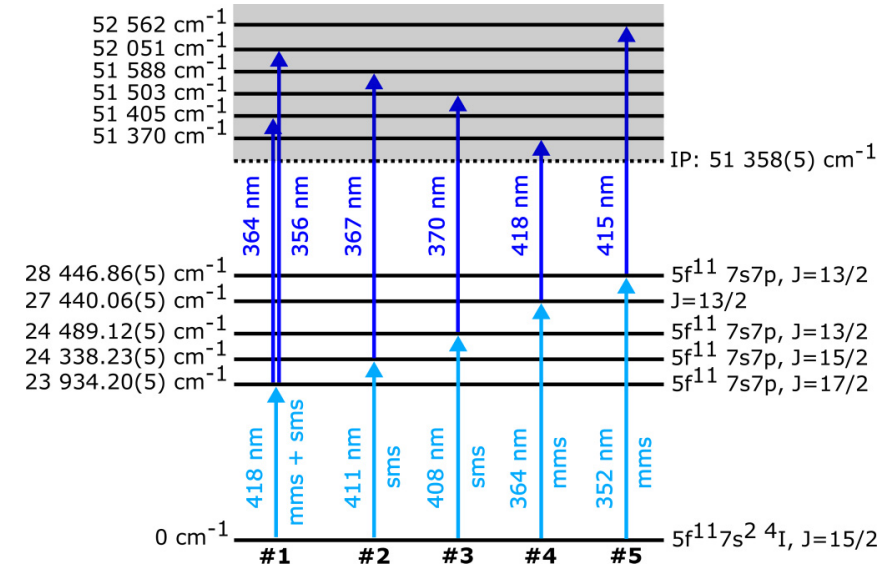

FIG. 3. Overview of the used laser ionization schemes with experimental level energies, configurations, and $J$ assignments. Five ground-state transitions were investigated for ${ }^{254}$ Es with multi-mode and single-mode spectroscopy ( $\mathrm{mms}$ and sms). The values for the IP and the electron configurations are taken from $[10,11,17,25]$.

placed in a tubular tantalum atomizer. Resistive heating produced an atomic einsteinium vapor inside the atomizer, which was probed by two-step RIS with the schemes shown in Fig. 3. The use of zirconium foils promotes the release of neutral atoms $[23,24]$. To obtain low background rates, the atomizer was preconditioned by baking it under vacuum conditions at about $2000 \mathrm{~K}$ for several hours before inserting the sample. Einsteinium atoms were released from the zirconium foil at temperatures of $700-1300{ }^{\circ} \mathrm{C}$, similar to experiments reported in [18]. The ions were extracted, accelerated to $30 \mathrm{keV}$, and guided towards a dipole magnet separating them according to their mass-to-charge ratio. Ions passing the separator slit, placed in the focal plane of the magnet, are detected with a secondary electron multiplier.

The laser system consisted of three ns-Ti:Sa lasers, pumped by two frequency-doubled Nd:YAG lasers $(10 \mathrm{kHz}$ repetition rate). Two Ti:Sa lasers were equipped with a grating as a frequency-selective element and featured an internal second harmonic generation (SHG) unit. For multi-mode hyperfine spectroscopy (mms) these lasers were equipped with an etalon, which reduced the bandwidth to 2-3 GHz. The third laser was an injection-locked Ti:Sa laser, seeded by a continuous-wave (cw) external-cavity diode laser and equipped with an external single-pass SHG unit. This laser features a bandwidth of only $20 \mathrm{MHz}$, but the accessible wavelength range was limited to that available by the $\mathrm{cw}$ master laser [26,27]. This was therefore used only for selected transitions (single-mode spectroscopy, sms). The laser pulse length was about $40 \mathrm{~ns}$, and the average output power was $100-600 \mathrm{~mW}$. Pulse synchronization was achieved by external triggering of the pump lasers with a pulse generator. After optimization of the lasers and the identification of autoionizing resonances above the IP for highest ionization efficiency, five different ground-state transitions were investigated in detail. Here, the $352 \mathrm{~nm}$-ground-state transition was probed in ${ }^{253,254,255}$ Es, while the other transitions were studied only in ${ }^{254}$ Es. 

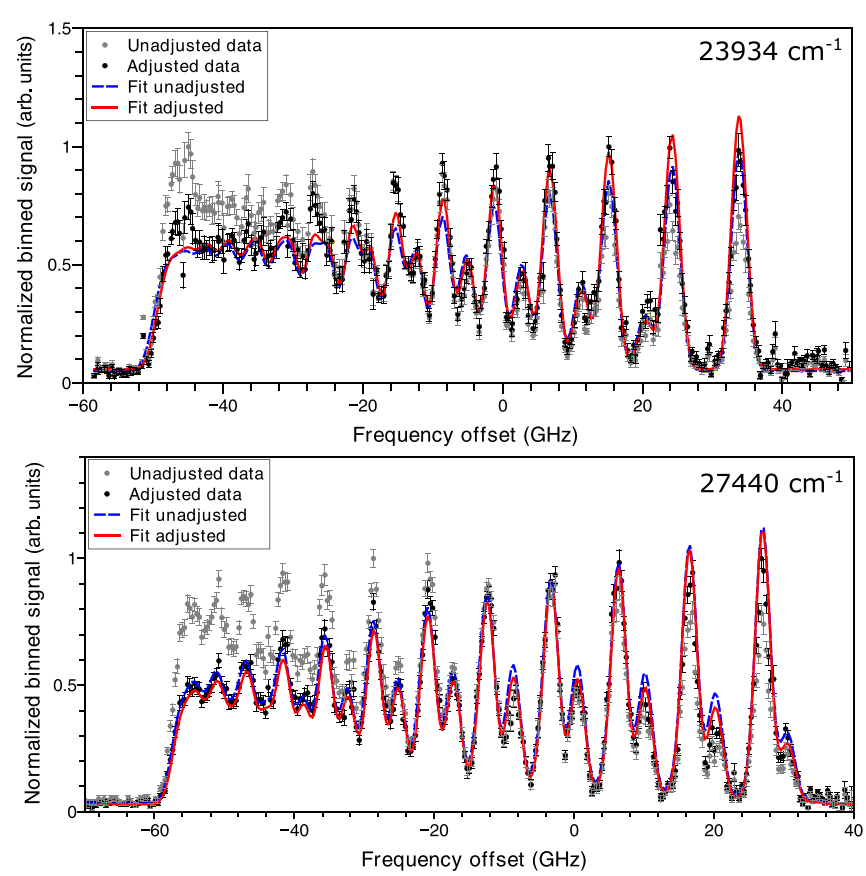

FIG. 4. Measured hyperfine spectrum of the transition from the ground-state to the $23934.20(5) \mathrm{cm}^{-1}$ (top) and $27440.06(5) \mathrm{cm}^{-1}$ (bottom) states, showing the measured data (gray points) and the fit (blue dashed line). Data resulting after subtracting a linear background (black points) and the fit (solid red line) are shown.

The spectral resolution is affected by the power of the spectroscopy laser and by the synchronicity of laser pulses for the first and second transition [28]. The laser power and the delay of the second step laser pulse were chosen for each
TABLE I. Overview over the ground-state $A$ and $B$ hyperfine parameters (in $\mathrm{MHz}$ ) extracted from the adjusted and unadjusted data for a spin of $I=7$.

\begin{tabular}{lccccc}
\hline \hline & \multicolumn{2}{c}{ Level at $23934.20(5) \mathrm{cm}^{-1}$} & & \multicolumn{2}{c}{ Level at 27 $440.06(5) \mathrm{cm}^{-1}$} \\
\cline { 2 - 3 } \cline { 5 - 6 } & Unadjusted & Adjusted & & Unadjusted & Adjusted \\
\hline$A_{\mathrm{gs}}$ & $355.4(10.8)$ & $348.0(11.4)$ & & $340.9(10.4)$ & $342.4(9.5)$ \\
$B_{\mathrm{gs}}$ & $-6177(923)$ & $-5887(1015)$ & & $-6721(713)$ & $-6577(772)$ \\
rel. $\chi^{2}$ & 2.76 & 1.00 & & 4.44 & 1.00 \\
\hline \hline
\end{tabular}

transition for highest spectral resolution without compromising the total efficiency. The signal events were recorded in dependence of the wavelength of the first excitation laser, measured by commercial wavelength meters (High Finesse, WS7 for mms, WSU-30 for sms). The obtained spectra were fitted using the SATLAS package, considering saturation for the peak intensities [29]. Due to the dominant contribution from Doppler broadening (and the laser bandwidth in the case of mms) the obtained spectra are well described by Gaussian fit profiles (FWHM typically $2.5 \mathrm{GHz}$ for mms and $1.5 \mathrm{GHz}$ for sms). For the transitions to the $23934 \mathrm{~cm}^{-1}$ and the $27440 \mathrm{~cm}^{-1}$ levels, an additional trend was observed in the spectra depending on the laser frequency, which could be related to a change in evaporation rate with temperature or due to wavelength-dependent laser power fluctuations. To model this trend a linear function was subtracted from the measured data points, see Fig. 4. Although the extracted hyperfine structure parameters $A$ and $B$ for the adjusted and non adjusted data coincide (cf. Table I), only this adjustment allowed fitting all individually measured hyperfine structures

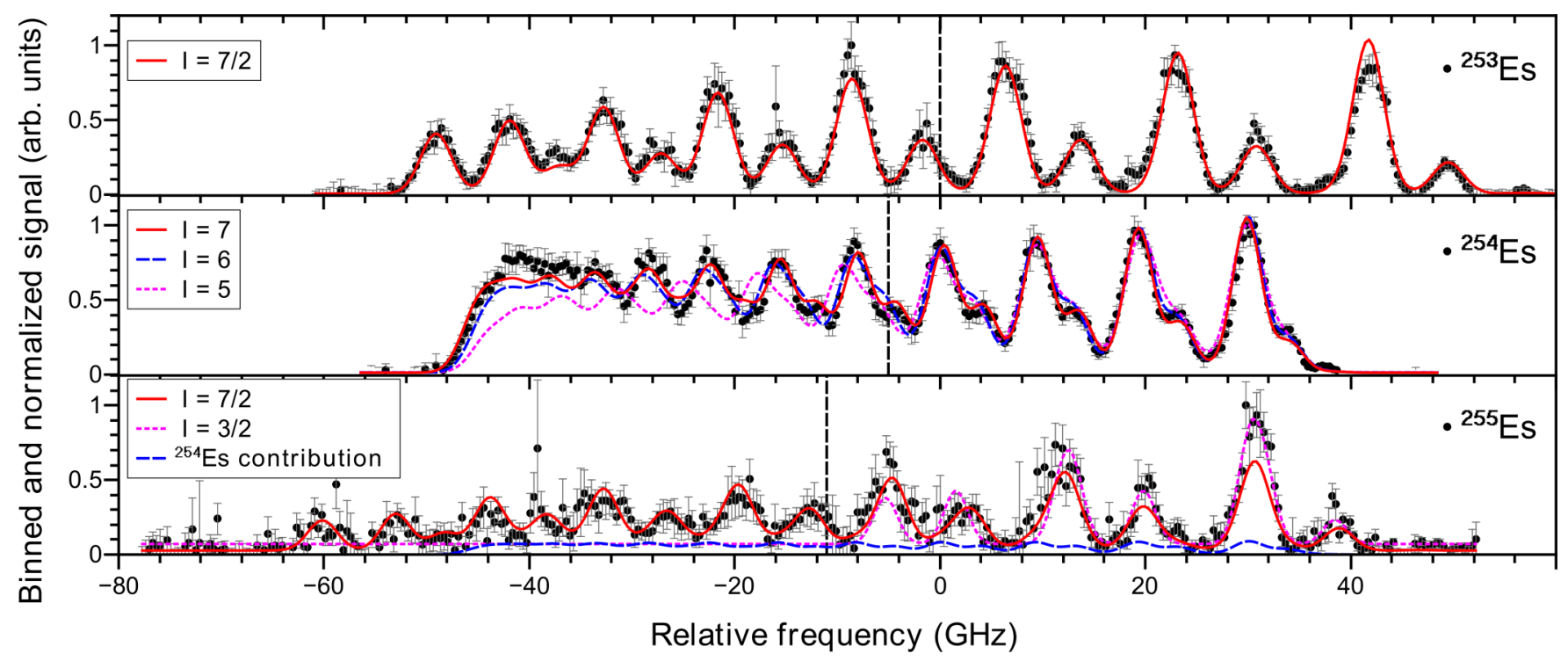

FIG. 5. Measured hyperfine spectra for the ground-state transition to the $28447 \mathrm{~cm}^{-1}$ state (\#5 in Fig. 3). The centroid frequency $v^{253}$ of the ${ }^{253}$ Es hyperfine structure is taken as reference and the centroids are indicated by the black dashed lines. The colored lines represent the best fits for each isotope to the data points for different values of the nuclear spin. The fit parameters are given in Table II. Due to the low abundance of ${ }^{255} \mathrm{Es}$, the sample was heated to higher temperatures leading to a decrease of the signal in the scanning range from right to left. There is a small contribution from ${ }^{254}$ Es to the signal, because a small fraction of ${ }^{254}$ Es reached the detector when the magnet was set to $A / q=255$ ions. 
TABLE II. Overview over the measured energy levels of Es (in $\mathrm{cm}^{-1}$ ) and extracted hyperfine constants (in MHz) obtained from groundstate transitions.

\begin{tabular}{|c|c|c|c|c|c|c|c|c|c|}
\hline \multirow[b]{2}{*}{$J$} & \multirow[b]{2}{*}{$E_{\text {exp }}$} & \multicolumn{4}{|c|}{${ }^{253} \mathrm{Es}$} & \multicolumn{2}{|c|}{${ }^{254}$ Es } & \multicolumn{2}{|c|}{${ }^{255}$ Es } \\
\hline & & $A_{\text {exp }}$ & $A_{\text {lit }}$ & $B_{\exp }$ & $B_{\text {lit }}$ & $A_{\text {exp }}$ & $B_{\exp }$ & $A_{\text {exp }}$ & $B_{\exp }$ \\
\hline $17 / 2$ & $23934.20(5)$ & & $(2284(15))^{\mathrm{a}^{\dagger}}$ & & & $951(8)$ & $-6930(690)$ & & \\
\hline $15 / 2$ & $24338.23(5)$ & & $(1532(15))^{\mathrm{a} \dagger}$ & & & $626(5)$ & $-11120(200)$ & & \\
\hline $13 / 2$ & $24489.12(5)$ & & $(99(15))^{\mathrm{a}^{\dagger}}$ & & & $28(8)$ & $-12945(440)$ & & \\
\hline
\end{tabular}

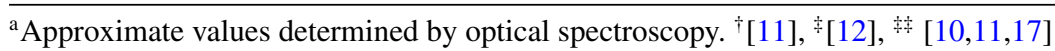

for ${ }^{254}$ Es simultaneously with shared ground-state hyperfine parameters $A_{\mathrm{gs}}$ and $B_{\mathrm{gs}}$ in a combined fit.

Experimental results. Figure 5 shows the measured hyperfine spectra for the $352 \mathrm{~nm}$-ground-state transition in ${ }^{253,254,255}$ Es. Albeit the presence of ${ }^{255}$ Es was not expected based on the initial analysis by $\alpha$ spectroscopy, it was observed at about 1000-fold reduced abundance compared with ${ }^{254} \mathrm{Es}$. Due to the finite mass-resolving power of the RISIKO dipole magnet $(R \approx 1000[20]),{ }^{254}$ Es contributed $9(2) \%$ to the signal at mass number 255 (dashed line in the lower panel of Fig. 5). For ${ }^{253} \mathrm{Es}$, the nuclear spin is known to be $I\left({ }^{253} \mathrm{Es}\right)=7 / 2^{+}[30]$ with an unpaired proton configuration of $\pi_{7 / 2}$ [633]. The ground-state hyperfine parameters extracted from our fit (upper panel in Fig. 5) are $A=802$ (18) $\mathrm{MHz}$ and $B=-3916(550) \mathrm{MHz}$ and agree with the ABMR measurements [12].

In the literature the nuclear spins of ${ }^{254,255}$ Es are tentatively assigned to $I\left({ }^{254} \mathrm{Es}\right)=(7)$ and $I\left({ }^{255} \mathrm{Es}\right)=(7 / 2)$ [31,32]. For ${ }^{255}$ Es with one unpaired proton, the nuclear spin was assumed based on a systematic trend along the Es isotopes and Nilsson-model calculations giving a $\pi_{7 / 2}$ [633] ground-state configuration [33]. The $\alpha$-decay scheme to ${ }^{251} \mathrm{Bk}$ does not conflict with this assignment [34]. Nevertheless, theoretical calculations predict the $\pi_{3 / 2}$ [521] orbital to be energetically higher but close-lying to the $\pi_{7 / 2}[633]$ orbital $[33,35]$. In ${ }^{251} \mathrm{Es}$, the ground-state spin is indeed confirmed as $3 / 2$ in contrast to a $7 / 2$ prediction from theory $[33,36]$. In general, by using the dependence of the hyperfine splitting on the nuclear spin $I$, an assignment of the nuclear spin is possible [5]. From the evaluation of our data for ${ }^{255} \mathrm{Es}$, it is obvious that neither a spin $1 / 2$ nor $3 / 2$ (dotted line in the lower panel of Fig. 5) can be taken for the fit to mimic the ${ }^{255}$ Es data, whereas assuming a spin of 7/2 (solid line) results in a good fit.

For ${ }^{254} \mathrm{Es}$, a former tentative spin assignment of $I\left({ }^{254} \mathrm{Es}\right)=$ (7) results from a coupling to maximum spin of the $\pi_{7 / 2}$ [633] proton configuration known to be the ground-state configuration in the neighboring isotopes ${ }^{253,255} \mathrm{Es}$ and the $\nu_{7 / 2}[613]$ neutron configuration of the isotone ${ }^{255} \mathrm{Fm}[32,37]$. This spin assignment is consistent with observed low $\beta^{-}$and electron capture decay branches to ${ }^{254} \mathrm{Fm}$ and ${ }^{254} \mathrm{Cf}$, respectively [13]. From the HFS fits to our ${ }^{254}$ Es data (see Fig. 5), a spin of $I\left({ }^{254} \mathrm{Es}\right)=7$ confirms former tentative findings. For lower spin values, the fit does not match with the observed HFS splitting (see middle panel of Fig. 5). For larger spin values, each individual transition (see Fig. 6) results in a proper fit, but the five individual transitions do not produce the same ground-state hyperfine parameters when $I \neq 7$ (see Fig. 7).

From the fit to the data, also the isotope shift $\delta v^{A_{0}, A_{1}}=$ $v^{A_{1}}-v^{A_{0}}$ from an isotope with mass number $A_{1}$ to a reference isotope with mass number $A_{0}$ was extracted for the ground-state transition to the $28447 \mathrm{~cm}^{-1}$ level. The measured shifts are $\delta v^{253,254}=-5.020(54) \mathrm{GHz}$ and $\delta v^{253,255}=$ $-11.046(86) \mathrm{GHz}$. The negative sign of the isotope shift agrees with the expectation for a $5 \mathrm{f}^{11} 7 \mathrm{~s}^{2} \rightarrow 5 \mathrm{f}^{11} 7 \mathrm{~s} 7 \mathrm{p}$ transition as assigned in [11], which reflects decreasing binding of the s-orbital for increasing nuclear volume.

In the heavy actinides, the atomic factors, which link isotope shifts with the change in nuclear volume, can usually only be obtained from atomic calculations $[5,16]$. Large electron correlations of open $f$-shell systems complicate these calculations. To date, einsteinium remains inaccessible for atomic theory, whereas recently calculations for the next heavier element, fermium, became available [38], indicating that further progress can be expected in the near future. Assuming a negligible contribution from the mass shift, a very small non-inverted odd-even staggering is seen in the

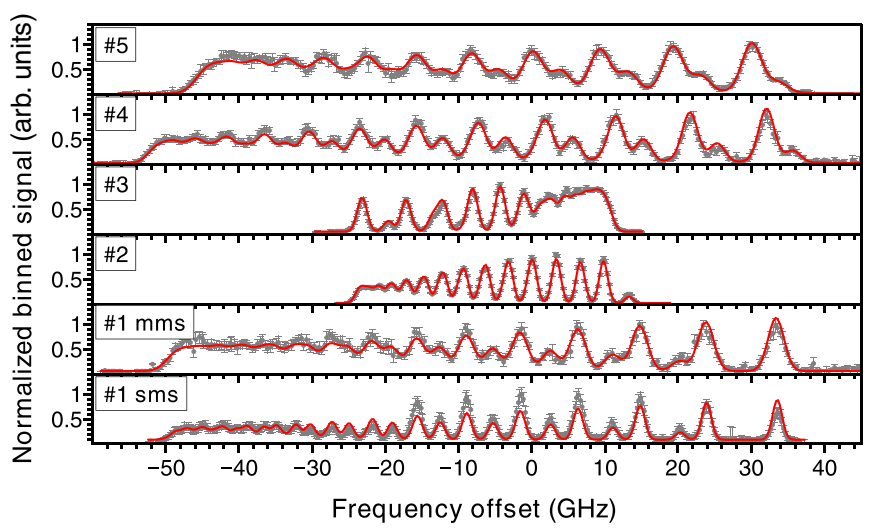

FIG. 6. Overview of all measured ground-state transitions in ${ }^{254} \mathrm{Es}$ as referenced in Fig. 3. The frequency offset to the center of gravity in $\mathrm{GHz}$ is shown for each transition. The fit (red lines) was performed using the same ground-state hyperfine coupling constants $A$ and $B$ for all transitions. The spectra for $23934 \mathrm{~cm}^{-1}$ in mms and $27440 \mathrm{~cm}^{-1}$ in mms were adjusted prior to fitting as described in the text. 


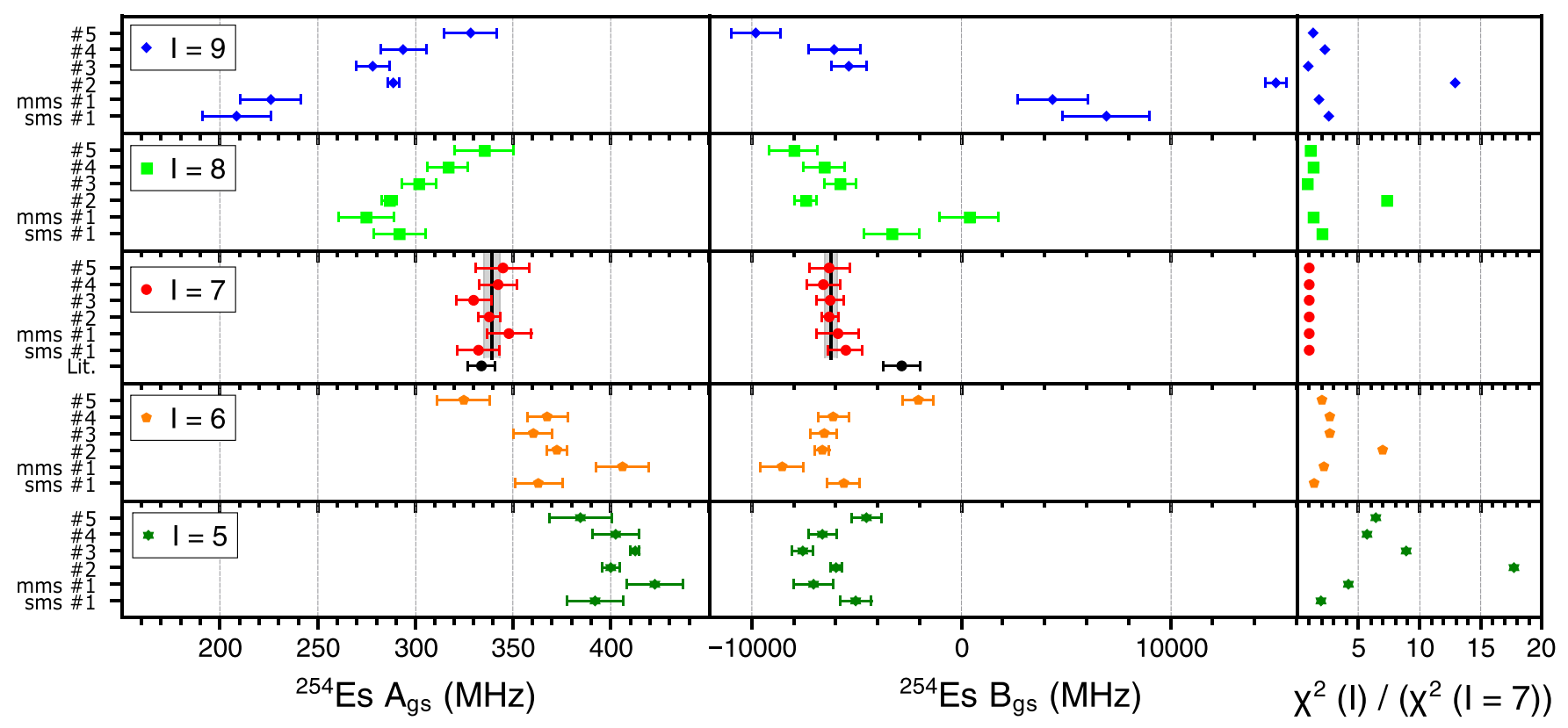

FIG. 7. Overview of the individual ground-state HFS parameters $A$ (left) and $B$ (middle) in ${ }^{254}$ Es with their total uncertainties as referenced in Fig. 3 together with literature values (black circles) [18]. The $A$ factor matches with our findings. The discrepancy in the $B$ factor may be due to insufficient statistics and limited resolution in the measurements on a single transition in [18]. Errors according to the Birge ratio are given as discussed in text. The solid lines mark the final values (weighted average) with uncertainties as indicated by the gray shaded area. The ratio $\chi^{2}(I) /\left(\chi^{2}(I=7)\right)$ (right) quantifies the quality of each fit compared to the fit for $I=7$.

measured einsteinium data, which is indicated by the larger isotope shift between ${ }^{254,255}$ Es compared to ${ }^{253,254}$ Es. With the obtained spin values, the HFS constants $A$ and $B$ were determined from the ground-state transitions and are summarized in Table II. For ${ }^{253}$ Es, the ground-state parameters are known with high precision from the ABMR measurements. HFS parameters for selected excited levels are also reported from optical spectroscopy [10], but the large uncertainty given for the $B$-parameter made it difficult, to fix the ratio of the excited state and ground-state (gs) HFS constants in our evaluation. An overview of the extracted parameters for each of the five transitions is shown in Fig. 7. The error bars (1 sigma) were obtained from the fit error. Due to the scatter of the individual ground-state parameters the fit uncertainties were increased for all extracted hyperfine parameters by a factor of three ( $A$-factors) and two ( $B$-factors) analyzing the respective Birge ratio $[39,40]$, which is displayed in Fig. 7. From the adjusted individual ground-state parameters a weighted average (solid black line) with uncertainty (gray shaded area) was calculated. The magnetic dipole moments and the spectroscopic electric quadrupole moments for ${ }^{254,255}$ Es were determined from the hyperfine parameters obtained for the ground-state using the relations

$$
\mu_{I}=\frac{A}{A_{\text {ref }}} \frac{I}{I_{\text {ref }}} \mu_{I, \text { ref }} \quad \text { and } \quad Q_{s}=\frac{B}{B_{\text {ref }}} Q_{s, \text { ref }}
$$

where the HFS parameters and the known moments of ${ }^{253}$ Es [12] serve as reference. The results are summarized in Table III together with the nuclear spins. The similar nuclear magnetic dipole moments of ${ }^{253}$ Es and ${ }^{255}$ Es indicate that the unpaired proton occupies the same single-particle orbital in both cases, pointing to a $\pi_{7 / 2}[633]$ configuration for the nuclear ground-state in ${ }^{255} \mathrm{Es}$. Our value for the magnetic dipole moment of ${ }^{254}$ Es is $3.42(7) \mu_{N}$ and deviates by about two sigma from the reported literature value of $\left|\mu_{I}\left({ }^{254} \mathrm{Es}\right)\right|=$ 4.35(41) $\mu_{N}$ [15]. Our measurements benefit from a large redundancy in the determination of the HFS parameters from individual transitions and do not rely on external magnetic fields from the host lattice, which have to be calibrated independently. Furthermore our value agrees rather well with a magnetic moment of $\left|\mu_{I}\left({ }^{254} \mathrm{Es}\right)\right|=3.52 \mu_{N}$ calculated for the assumed $\pi_{7 / 2}[633] \nu_{7 / 2}[613]$ configuration [13] in the same work [15] using a single-particle model [41]. In addition, the ratio of the hyperfine parameters of ground and excited states is constant and lies well within the uncertainty bands and thus, a contribution from hyperfine anomaly [42] can be excluded. The spectroscopic electric quadrupole moment $Q_{s}$ for ${ }^{254} \mathrm{Es}$ is $9.6(1.2) e \mathrm{~b}$, i.e. considerably larger than in

TABLE III. Overview over the extracted nuclear magnetic and electric quadrupole moments of Es isotopes in comparison to the literature.

\begin{tabular}{lccclcc}
\hline \hline & & \multicolumn{2}{c}{ This work } & & \multicolumn{2}{c}{ Literature } \\
\cline { 5 - 6 } Isotope & $I$ & $\mu_{I}\left(\mu_{N}\right)$ & $Q_{s}(e \mathrm{~b})$ & & $\mu_{I}\left(\mu_{N}\right)$ & $Q_{s}(e \mathrm{~b})$ \\
\hline${ }^{253} \mathrm{Es}$ & $7 / 2$ & & & & $4.10(7)[12]$ & $6.7(8)^{\mathrm{a}}[12]$ \\
${ }^{254} \mathrm{Es}$ & 7 & $3.42(7)$ & $9.6(1.2)$ & & $4.35(41)[15]$ & \\
${ }^{254 \mathrm{~m}} \mathrm{Es}$ & 2 & & & & $2.90(7)[12]$ & $3.8(5)[12]$ \\
${ }^{255} \mathrm{Es}$ & $7 / 2$ & $4.14(10)$ & $5.1(1.7)$ & & \\
\end{tabular}

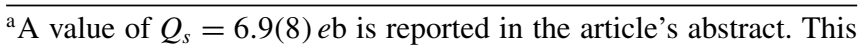
differs from the value in the main article. 
${ }^{253}$ Es. Calculating the intrinsic quadrupole moment $Q=(I+$ 1) $(2 I+3) /\left(3 K^{2}-I(I+1)\right) \cdot Q_{s}$ with $K=I$ for the groundstate as band head results in $Q\left({ }^{253} \mathrm{Es}\right)=14.4(1.7) \mathrm{eb}$ and $Q\left({ }^{254} \mathrm{Es}\right)=14.3(1.8) \mathrm{eb}$, which indicates a similar deformation for the heavier isotope. For ${ }^{255}$ Es the value of $Q\left({ }^{255} \mathrm{Es}\right)=$ $10.9(3.6) e b$ is in agreement with an expected drop in deformation after passing the deformed shell closure at $N=152$.

Summary. Electromagnetic moments in ${ }^{253-255}$ Es, located well above the deformed $N=152$ shell closure were obtained using laser spectroscopy. From the measured spectra the hyperfine constants were deduced. The magnetic dipole moments and electric quadrupole moments for ${ }^{254,255}$ Es were extracted. The magnetic moment for ${ }^{255} \mathrm{Es}$ and the quadrupole moments for ${ }^{254,255}$ Es were experimentally determined for the first time. Our value for the magnetic dipole moment of ${ }^{254} \mathrm{Es}$ is more precise than the value from Ref. [15], with the two deviating by about two sigma for the latter. The quadrupole deformation around $N=152$ was shown to be similar for ${ }^{253}$ Es and ${ }^{254}$ Es within uncertainties, while a hint of a decrease in deformation towards a more spherical nuclear shape is observed in ${ }^{255}$ Es. On top of that, the isotope shifts for
${ }^{253-255}$ Es in one ground-state transition were extracted. The availability of theory input for the field shift and mass shift parameter for this transition will make calculations of the changes in the mean-square charge radii possible. Our data motivate further developments in atomic theory, which is very demanding for many-electron systems such as einsteinium with its 11 electrons in the $f$-shell. Despite low sample size, laser spectroscopic studies of ${ }^{255} \mathrm{Es}$ were possible, indicating a sufficient sensitivity for detailed laser spectroscopy of the next heavier and even rarer element fermium.

Acknowledgments. This research was supported by the U.S. Department of Energy, Office of Science, Office of Basic Energy Sciences, Heavy Elements Chemistry Program, under Award DE-FG02-13ER16414. The isotopes used in this research were supplied by the U.S. DOE Isotope Program, managed by the Office of Science. This work has been supported by the Bundesministerium für Bildung und Forschung (BMBF, Germany) under Project No. 05P18UMCIA. This project has received funding from the European Union's Horizon 2020 research and innovation programme under grant agreement No 861198-LISA-H2020-MSCA-ITN-2019.
[1] E. Minaya Ramirez, D. Ackermann, K. Blaum, M. Block, C. Droese, Ch. E. Düllmann, M. Dworschak, M. Eibach, S. Eliseev, E. Haettner, F. Herfurth, F. P. Hessberger, S. Hofmann, J. Ketelaer, G. Marx, M. Mazzocco, D. Nesterenko, Y. N. Novikov, W. R. Plass, D. Rodriguez et al., Science 337, 1207 (2012).

[2] Z. Patyk and A. Sobiczewski, Phys. Lett. B 256, 307 (1991).

[3] M. Block, Radiochim. Acta 107, 821 (2019).

[4] D. T. Yordanov, L. V. Rodríguez, D. L. Balabanski, J. Bieroń, M. L. Bissell, K. Blaum, B. Cheal, J. Ekman, G. Gaigalas, R. F. G. Ruiz, G. Georgiev, W. Gins, M. R. Godefroid, C. Gorges, Z. Harman, H. Heylen, P. Jönsson, A. Kanellakopoulos, S. Kaufmann, C. H. Keitel et al., Commun. Phys. 3, 1 (2020).

[5] P. Campbell, I. D. Moore, and M. R. Pearson, Prog. Part. Nucl. Phys. 86, 127 (2016).

[6] J. B. Roberto, C. W. Alexander, R. A. Boll, J. D. Burns, J. G. Ezold, L. K. Felker, S. L. Hogle, and K. P. Rykaczewski, Nucl. Phys. A 944, 99 (2015).

[7] S. M. Robinson, D. E. Benker, E. D. Collins, J. G. Ezold, J. R. Garrison, and S. L. Hogle, Radiochim. Acta 108, 737 (2020).

[8] S. Raeder, D. Ackermann, H. Backe, R. Beerwerth, J. C. Berengut, M. Block, A. Borschevsky, B. Cheal, P. Chhetri, Ch. E. Düllmann, V. A. Dzuba, E. Eliav, J. Even, R. Ferrer, V. V. Flambaum, S. Fritzsche, F. Giacoppo, S. Götz, F. P. Heßberger, M. Huyse et al., Phys. Rev. Lett. 120, 232503 (2018).

[9] W. S. Aaron, C. W. Alexander, R. L. Cline, E. D. Collins, J. A. Klein, J. B. Knauer, and S. Mirzadeh, in Office of Scientific \& Technical Information Technical Reports (Oak Ridge National Laboratory (ORNL), Oak Ridge, TN, United States, 1999).

[10] E. F. Worden, R. W. Lougheed, R. G. Gutmacher, and J. G. Conway, J. Opt. Soc. Am. 64, 77 (1974).

[11] J.-F. Wyart, J. Blaise, and E. F. Worden, J. Solid State Chem. 178, 589 (2005).
[12] L. S. Goodman, H. Diamond, and H. E. Stanton, Phys. Rev. A 11, 499 (1975).

[13] B. Singh, Nucl. Data Sheets 156, 1 (2019).

[14] G. M. Gurevich, A. L. Erzinkyan, and V. P. Parfenova, Bull. Russ. Acad. Sci.: Phys. 75, 520 (2011).

[15] N. Severijns, A. A. Belyaev, A. L. Erzinkyan, P. D. Eversheim, V. T. Filimonov, V. V. Golovko, G. M. Gurevich, P. Herzog, I. S. Kraev, A. A. Lukhanin, V. I. Noga, V. P. Parfenova, T. Phalet, A. V. Rusakov, M. Tandecki, Y. G. Toporov, C. Tramm, E. Traykov, S. VanGorp, V. N. Vyachin et al., Phys. Rev. C 79, 064322 (2009).

[16] M. Block, M. Laatiaoui, and S. Raeder, Prog. Part. Nucl. Phys. 116, 103834 (2021).

[17] J. Blaise and J. F. Wyart, Constantes selectionnees niveaux d'energie et spectres atomiques des actinides (Centre National de la Recherche Scientifique, Paris, France, 480, Paris, 1992).

[18] M. Nunnemann, Ph.D. thesis, Institut für Physik, Johannes Gutenberg-Universität Mainz, 1999.

[19] T. Kieck, S. Biebricher, Ch. E. Düllmann, and K. Wendt, Rev. Sci. Instrum. 90, 053304 (2019).

[20] T. Kron, Y. Liu, S. Richter, F. Schneider, and K. Wendt, J. Phys. B 49, 185003 (2016).

[21] T. Kieck, H. Dorrer, Ch. E. Düllmann, V. Gadelshin, F. Schneider, and K. Wendt, Nucl. Instrum. Methods. A 945, 162602 (2019).

[22] S. Hogle, C. W. Alexander, J. D. Burns, J. G. Ezold, and G. I. Maldonado, Nucl. Sci. Eng. 185, 473 (2017).

[23] B. Eichler, S. Hübener, N. Erdmann, K. Eberhardt, H. Funk, G. Herrmann, S. Köhler, N. Trautmann, G. Passler, and F.-J. Urban, Radiochim. Acta 79, 221 (1997).

[24] K. Zhang, D. Studer, F. Weber, V. M. Gadelshin, N. Kneip, S. Raeder, D. Budker, K. Wendt, T. Kieck, S. G. Porsev, C. Cheung, M. S. Safronova, and M. G. Kozlov, Phys. Rev. Lett. 125, 073001 (2020). 
[25] N. Erdmann, M. Nunnemann, K. Eberhardt, G. Herrmann, G. Huber, S. Koehler, J. V. Kratz, G. Passler, J. R. Peterson, N. Trautmann, and A. Waldek, J. Alloy. Compd. 271, 837 (1998).

[26] T. Kessler, H. Tomita, C. Mattolat, S. Raeder, and K. Wendt, Laser Phys. 18, 842 (2008).

[27] V. Sonnenschein, I. D. Moore, S. Raeder, M. Reponen, H. Tomita, and K. Wendt, Laser Phys. 27, 085701 (2017).

[28] R. P. de Groote, I. Budinčević, J. Billowes, M. L. Bissell, T. E. Cocolios, G. J. Farooq-Smith, V. N. Fedosseev, K. T. Flanagan, S. Franchoo, R. F. Garcia Ruiz, H. Heylen, R. Li, K. M. Lynch, B. A. Marsh, G. Neyens, R. E. Rossel, S. Rothe, H. H. Stroke, K. D. A. Wendt, S. G. Wilkins et al., Phys. Rev. Lett. 115, 132501 (2015).

[29] W. Gins, R. P. de Groote, M. L. Bissell, C. G. Buitrago, R. Ferrer, K. M. Lynch, G. Neyens, and S. Sels, Comp. Phys. Commun. 222, 286 (2018).

[30] E. F. Worden, R. G. Gutmacher, R. W. Lougheed, J. G. Conway, and R. J. Mehlhorn, J. Opt. Soc. Am. 60, 1297 (1970).

[31] K. J. Moody, R. W. Lougheed, J. F. Wild, R. J. Dougan, E. K. Hulet, R. W. Hoff, C. M. Henderson, R. J. Dupzyk, R. L. Hahn, K. Sümmerer, G. D. O'Kelley, and G. R. Bethune, Nucl. Phys. A 563, 21 (1993).
[32] E. Browne and J. K. Tuli, Nucl. Data Sheets 114, 1041 (2013).

[33] S. Ćwiok, S. Hofmann, and W. Nazarewicz, Nucl. Phys. A 573, 356 (1994).

[34] B. Buck, A. C. Merchant, and S. M. Perez, J. Phys. G 18, 143 (1992).

[35] A. Parkhomenko and A. Sobiczewski, Acta Phys. Pol. B35, 2447 (2004).

[36] I. Ahmad, R. K. Sjoblom, R. F. Barnes, E. P. Horwitz, and P. R. Fields, Nucl. Phys. A 140, 141 (1970).

[37] M. Asai, K. Tsukada, M. Sakama, H. Haba, T. Ichikawa, Y. Ishii, A. Toyoshima, T. Ishii, I. Nishinaka, Y. Nagame, Y. Kasamatsu, M. Shibata, Y. Kojima, and H. Hayashi, Phys. Rev. C 87, 014332 (2013).

[38] S. O. Allehabi, J. Li, V. A. Dzuba, and V. V. Flambaum, J. Quant. Spectrosc. Radiat. Transfer 253, 107137 (2020).

[39] R. T. Birge, Rev. Mod. Phys. 1, 1 (1929).

[40] O. Bodnar and C. Elster, Metrologia 51, 516 (2014).

[41] C.-D. Herrmann, B. Prillwitz, V. Dämmrich, K. Freitag, P. Herzog, D. Mayer, K. Schlösser, and I. Ragnarsson, Nucl. Phys. A 493, 83 (1989).

[42] S. Büttgenbach, Hyperfine Interact. 20, 1 (1984). 